\title{
Testing the Tester: Lessons Learned During the Testing of a State-of-the-Art Commercial 14nm Processor Under Proton Irradiation
}

\author{
Carl M. Szabo, Jr. \\ AS\&D, Inc., supporting \\ NASA Electronic Parts and Packaging (NEPP) Program \\ NASA/GSFC \\ carl.m.szabo@nasa.gov \\ 301-286-8890
}

Adam R. Duncan, Naval Surface Warfare Center Crane, and

Kenneth A. LaBel, NASA GSFC 


\section{Acronyms}

- Basic Input/Output System (BIOS)

- Device Under Test (DUT)

- Graphical Processing Unit (GPU)

- Goddard Space Flight Center (GSFC)

- High Definition Multimedia Interface (HDMI)

- Massachusetts General Hospital (MGH)

- National Aeronautics and Space Administration (NASA)

- NASA Electronic Parts and Packaging (NEPP) Program

- Random Access Memory (RAM)

- Solid State Disk (SSD)

- single event effect (SEE)

- Thermal Design Power (TDP)

- Universal Serial Bus (USB) 


\section{Background}

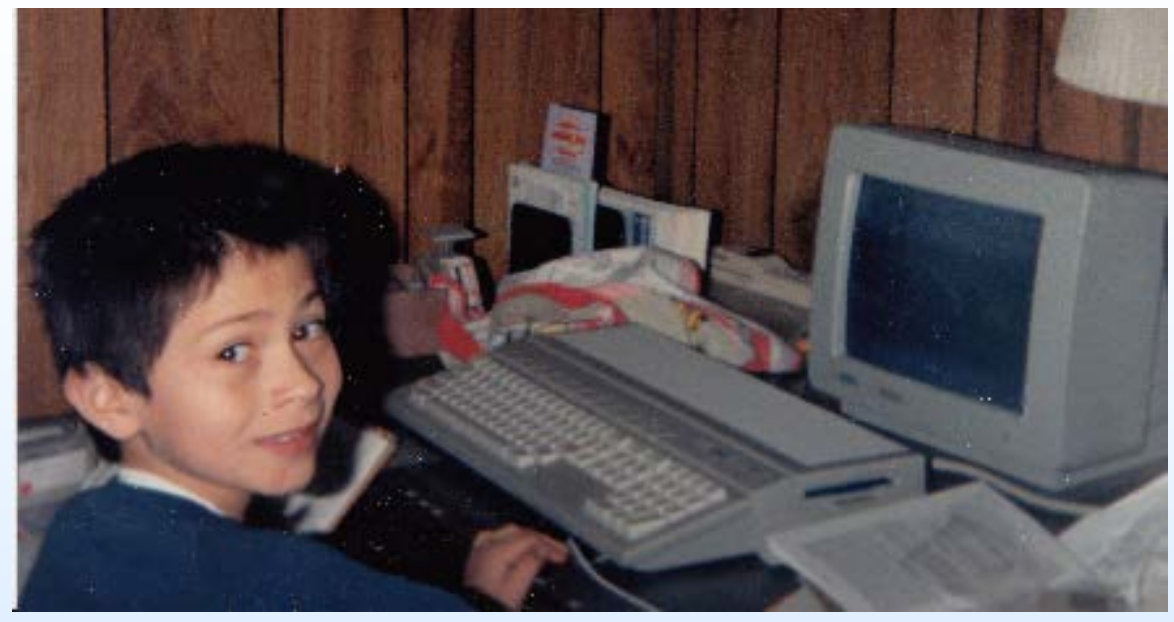

Circa 1986, playing with an Atari 1040ST

- Lifelong Computer Hobbyist and Enthusiast

- Unconventional Training and Skill Set

- System Administrator supporting GSFC since 2002

- Duties often require flexibility and out of the box thinking to solve unplanned problems I handle unexpected events

- Introduced to Radiation Effects $\sim 2012$

- "Person Under Test" 


\section{Device Under Test}

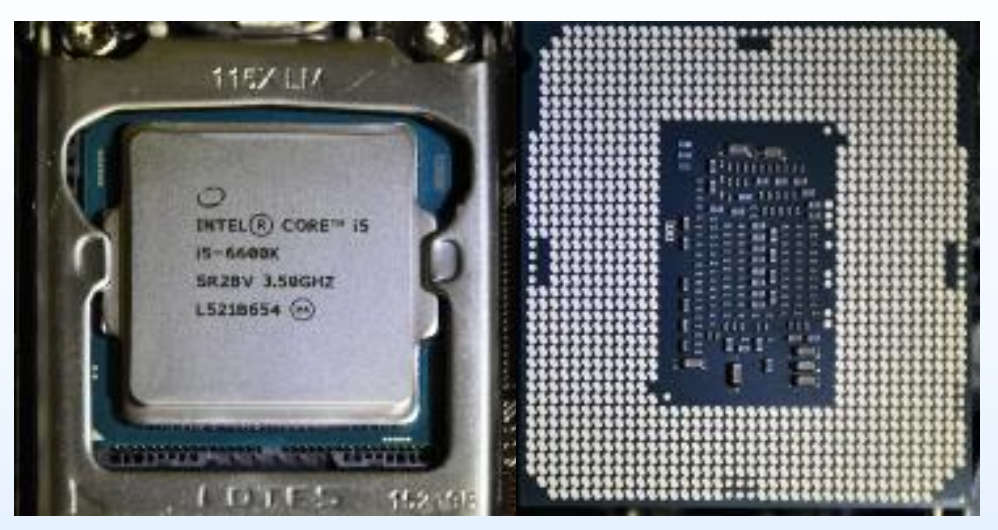

- Intel core i5-6600K "Skylake" Microprocessor

- ASUS Z170M-Plus Motherboard, 8GB RAM, 750W Power Supply, SSD, USB and HDMI over Ethernet control

- Microsoft Windows Server 2012R2 OS, HWiNFO System Monitoring, Linpack, FurMark, Prime95 Stress, Batch File Control

- In-situ, "System Level" Best Effort Approach

- Proton Testing via

- TRIUMF 105 MeV Beam Line (November 2015)

- MGH 200 MeV Beam Line (October 2016) 


\section{What Happened}

- Hard failure event observed during TRIUMF visit

- Device appeared to lose integrated GPU functionality during irradiation

- Failure occurred during "Full" test (Linpack + FurMark) with only $1 \mathrm{CPU}$ core active

- Results were difficult to explain at the time of testing

- Subsequent testing at MGH yielded no functional failures after $60+$ test runs!

- How??

- Next day at MGH, re-tested board used during TRIUMF tests

- Processors began to fail! 


\section{Troubleshooting 1/3}

- Product was new and period of testing was short

- First setup featured early release hardware

- Public discovers flaws (Prime95 lockup issue)

- Test setup evolved as the device technology matured

- Later procured motherboards featured updates

- BIOS revision of board used at MGH operated DUT differently than board and BIOS version used at TRIUMF

- Supporting hardware and software enabled enhanced data collection

- Accurate data

- Evolution of test setup allowed insight that was not possible in early testing

- Retesting on the MGH and TRIUMF-tested boards showed same behaviors with fresh processors 


\section{Troubleshooting 2/3}

- Large differences in functional parameters

- Failures only occurred during exposure to protons

- These differences would likely be transparent to regular users
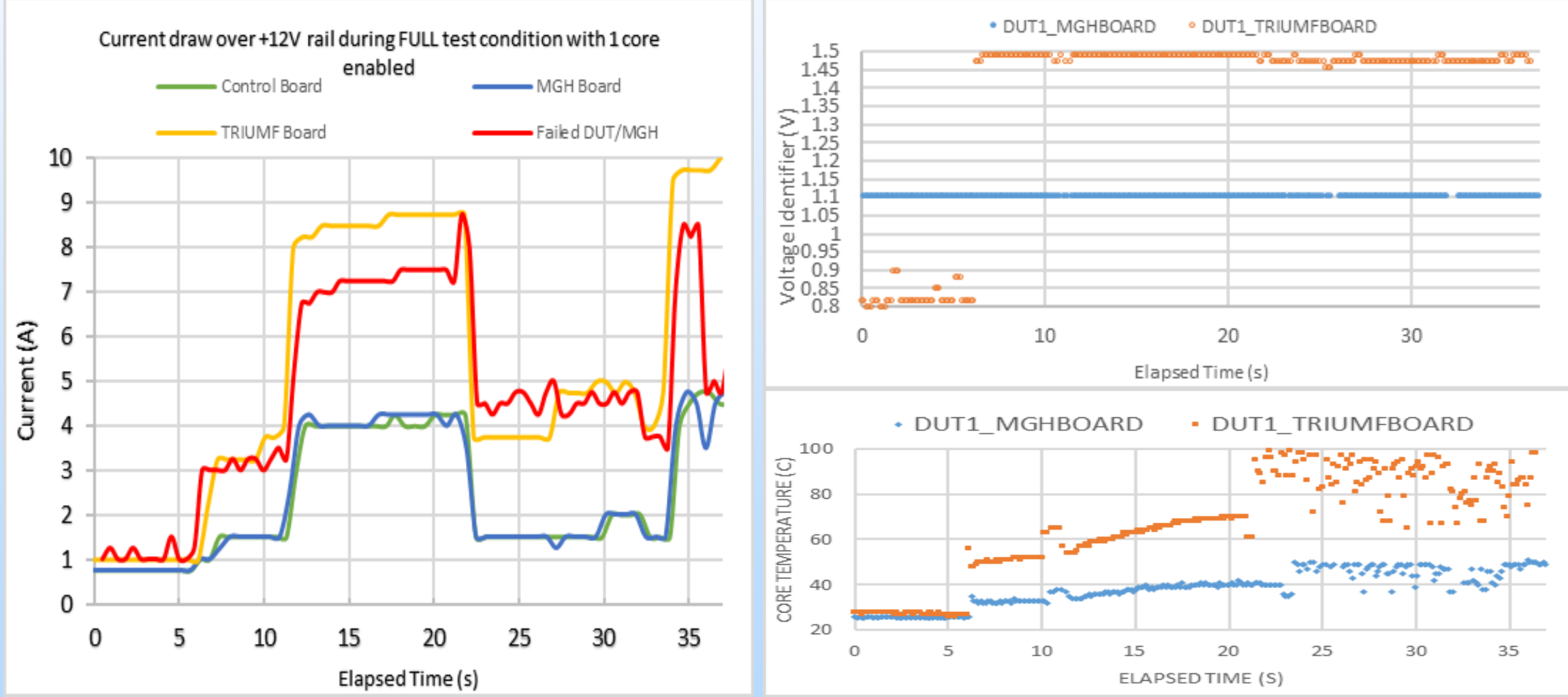


\section{Troubleshooting 3/3}

- Motherboard used at TRIUMF operated DUT in excess of rated 91 Watt TDP!

- Only 1 processing core active

- Degradation of performance after 18 hour extreme stress test

- Failed Linpack tests

- Could not reproduce GPU functional failure

- Motherboard used at MGH operated DUT more efficiently

- Lower temperature operation

- Fewer changes in voltage

- Slightly better performance

- Control Motherboard (latest BIOS available as of Sep. 2017)

- Behavior largely the same as MGH motherboard 


\section{Conclusion}

- Early hardware and software is imperfect

- Perform updates BIOS, microcode, hardware and software

- Up-to-date hardware and software leads to

- Increased data

- Accurate data

- Correctable I Uncorrectable Error Reporting

- However, current product cycle is changing quickly

- How feasible to characterize?

- Limited time begets limited reliability data

- Flight project cannot tolerate lack of supply + reliability data, nor frequent updates 